\title{
Associations and prognostic significance of diffuse myocardial fibrosis by cardiovascular magnetic resonance in heart failure with preserved ejection fraction
}

Clotilde Roy ${ }^{1,2^{*}}$ (D), Alisson Slimani ${ }^{1,2}$, Christophe de Meester ${ }^{1,2}$, Mihaela Amzulescu ${ }^{1,2}$, Agnes Pasquet ${ }^{1,2}$, David Vancraeynest ${ }^{1,2}$, Christophe Beauloye ${ }^{1,2}$, Jean-Louis Vanoverschelde ${ }^{1,2}$, Bernhard L. Gerber ${ }^{1,2}$ and Anne-Catherine Pouleur ${ }^{1,2}$

\begin{abstract}
Background: Increased myocardial fibrosis may play a key role in heart failure with preserved ejection fraction (HFpEF) pathophysiology. The study aim was to evaluate the presence, associations, and prognostic significance of diffuse fibrosis in HFpEF patients compared to age- and sex-matched controls.

Methods: We prospectively included 118 consecutive HFpEF patients. Diffuse myocardial fibrosis was estimated by extracellular volume (ECV) quantified by cardiovascular magnetic resonance with the modified Look-Locker inversion recovery sequence. We determined an ECV age- and sex-adjusted cutoff value (33\%) in 26 controls.

Results: Mean ECV was significantly higher in HFpEF patients versus healthy controls ( $32.9 \pm 4.8 \%$ vs $28.2 \pm 2.4 \%$, $P<0.001$ ). Multivariate logistic regression showed that body mass index (BMI) (odds ratio (OR) $=0.92$ [0.86-0.98], $P=0.011)$, diabetes $(\mathrm{OR}=2.62[1.11-6.18], P=0.028)$, and transmitral peak $E$ wave velocity $(\mathrm{OR}=1.02[1.00-1.03], P=$ 0.022 ) were significantly associated with abnormal ECV value. During a median follow-up of $11 \pm 6$ months, the primary outcome (all-cause mortality or first heart failure hospitalization) occurred in 38 patients. In multivariate Cox regression analysis, diabetes (hazard ratio $(H R)=1.98[1.04 ; 3.76], P=0.038)$ and hemoglobin level $(H R=0.81[0.67 ; 0.98], P=0.028)$ were significant predictors of composite outcome. The ECV ability to improve this model added significant prognostic information. We then developed a risk score including diabetes, hemoglobin and ECV > 33\% demonstrating significant prediction of risk and validated this score in a validation cohort of 53 patients. Kaplan-Meier curves showed a significant difference according to tertiles of the probability score $(P<0.001)$.
\end{abstract}

Conclusion: Among HFpEF patients, high ECV, likely reflecting abnormal diffuse myocardial fibrosis, was associated with a higher rate of all-cause death and first HF hospitalization in short term follow up.

Trial registration: Characterization of Heart Failure With Preserved Ejection Fraction. Trial registration number: NCT03197350. Date of registration: 20/06/2017. This trial was retrospectively registered.

Keywords: Diffuse myocardial fibrosis, Cardiac magnetic resonance, Prognosis

\footnotetext{
* Correspondence: clotilderoy23@gmail.com

'Division of Cardiology, Department of Cardiovascular Diseases, Cliniques

Universitaires St. Luc UCL, Av Hippocrate 10/2806, B-1200 Woluwé St.

Lambert, Belgium

${ }^{2}$ Pôle de Recherche Cardiovasculaire (CARD), Institut de Recherche

Expérimentale et Clinique (IREC), Université Catholique de Louvain, Brussels,

Belgium
}

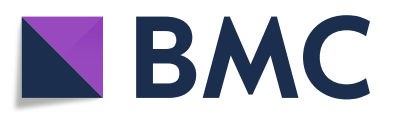

(c) The Author(s). 2018 Open Access This article is distributed under the terms of the Creative Commons Attribution 4.0 International License (http://creativecommons.org/licenses/by/4.0/), which permits unrestricted use, distribution, and reproduction in any medium, provided you give appropriate credit to the original author(s) and the source, provide a link to the Creative Commons license, and indicate if changes were made. The Creative Commons Public Domain Dedication waiver (http://creativecommons.org/publicdomain/zero/1.0/) applies to the data made available in this article, unless otherwise stated. 


\section{Background}

Heart failure (HF) with preserved ejection fraction (HFpEF) has been established as a major cause of cardiovascular morbidity and mortality, especially among the elderly $[1,2]$. Prevalence is increasing, affecting half of the patients with clinical signs of heart failure [3]. However, compared to HF with reduced ejection fraction (HFrEF), survival in HFpEF has not improved over time, and to date, no treatment effectively improves outcomes, probably because of the phenotypic heterogeneity of this syndrome [4].

Several mechanisms have been implicated in HFpEF, including advanced age and cardiovascular, metabolic, and pro-inflammatory comorbidities such as hypertension, diabetes, obesity, chronic obstructive pulmonary disease), coronary disease and renal failure [1, 4-6]. The exact pathophysiology of HFpEF remains unclear as a result of the absence of a proper animal model and the presence of numerous confounding effects. Recently, several studies using autopsies or myocardial biopsies have highlighted the key role of myocardial extracellular matrix abnormalities (fibrosis [7-9]) and myocardial structural changes such as altered cardiomyocyte function (hypertrophy [7]), systemic and coronary microvascular inflammation, and endothelial dysfunction (oxidative stress) as all being involved in myocardial stiffness and diastolic dysfunction [10, 11].

Quantification of extracellular volume fraction (ECV) by cardiovascular magnetic resonance (CMR) has recently emerged as a novel non-invasive diagnostic tool to assess myocardial fibrosis [12-14]. Recently, some studies have demonstrated the importance of diffuse or focal fibrosis estimated by biopsies or/and by CMR in patients with HFpEF $[15,16]$.

The aim of our study was to evaluate the presence, associations, and prognostic significance of ECV, likely reflecting diffuse myocardial fibrosis, in HFpEF patients.

\section{Methods}

\section{Study population}

Between December 2014 and October 2016, consecutive patients with suspected HFpEF were prospectively evaluated for inclusion in the study. The local ethics committee approved the study, and all patients gave written informed consent before study enrollment (Clinical trial NCT03197350).

The following criteria had to be fulfilled for study inclusion: New York Heart Association functional (NYHA) class $\geq \mathrm{II}$, typical signs of HF, NT-proBNP > $350 \mathrm{pg} / \mathrm{ml}$ and/or a hospitalization for $\mathrm{HF}$ in the previous 12 months, left ventricular (LV) ejection fraction (EF) $\geq 50 \%$, and relevant structural heart disease (LV hypertrophy/left atrial (LA) enlargement) and/or diastolic dysfunction by echocardiography [17]. Ischemic cardiomyopathy was defined as history of myocardial infarction or revascularization by either coronary artery bypass graft surgery (CABG) or coronary artery angioplasty. Diabetes was defined as an abnormal fasting glycaemia (>126 mg/dl) or the use of antidiabetic drugs.

The exclusion criteria were any contraindications to CMR (pacemaker, estimated glomerular filtration rate $(\mathrm{eGFR})<30 \mathrm{ml} / \mathrm{min} / \mathrm{m}^{2}$, claustrophobia), severe valvular disease, infiltrative (ie amyloidosis, sarcoidosis or hypertrophic cardiomyopathy, acute coronary syndrome in the last 30 days, chronic obstructive pulmonary disease GOLD 3 or 4, congenital heart disease, pericardial disease, atrial fibrillation with a ventricular response > $140 \mathrm{bpm}$, and severe anemia (hemoglobin $<7 \mathrm{~g} / \mathrm{dl}$ ). Patients with severe cognitive disorder were also excluded. One patient screened for the study was excluded due to presence of unknown cardiac amyloidosis.

Patients were compared to 26 age- and sex-matched controls without history of cardiovascular disease. Controls were recruited by advertisement in the community. All subjects underwent a full clinical exam, electrocardiogram (ECG), transthoracic echocardiography and exercise stress test, which all had to be normal prior to inclusion.

Controls and patients underwent blood sampling, complete transthoracic echocardiography and a CMR.

\section{Echocardiography}

All subjects underwent a two-dimensional (2D) transthoracic echocardiography at inclusion (iE33 system Philips Healthcare, Best, The Netherlands) with parasternal long- and short-axis views and apical views to assess LV and right ventricular (RV) systolic and diastolic functions and measurements of LA and right atrial (RA) volumes, as well as a valvular evaluation. Mitral valve inflow pattern ( $\mathrm{E}$ and $\mathrm{A}$ velocity) and septal and lateral mitral valve annular velocities (e') were recorded.

RV function was assessed by systolic annular tissue velocity of the lateral tricuspid annulus, tricuspid annular plane systolic excursion (TAPSE), and tracing the RV endocardium in the apical four-chamber view in systole and diastole to obtain fractional area change (RV FAC\%) [18]. All measurements were averaged over three beats in atrial fibrillation.

\section{Cardiovascular magnetic resonance}

CMR was performed using a 3 Tesla system (Ingenia, Philips Hearlthcare). Briefly, after localization of the heart, to assess LV and RV myocardial function and mass, 10-12 consecutive short-axis (SAX) images and 2-, 3-, and 4-chamber long-axis images of the LV were acquired using a cine balanced steady-state free precession sequence (bSSFP). Then, mid-ventricular short-axis 
modified Look-Locker inversion recovery (MOLLI) images were acquired for $\mathrm{T} 1$ determination using an 11-image, 18-heartbeat 3-(3)-3-(3)-5 bSSFP sequence. A total dose of $0.2 \mathrm{mmol} / \mathrm{kg}$ gadobutrol (Gadavist, Bayer Healthcare Leverkusen, Germany) was injected, and 1015 min after contrast injection, short- and long-axis 2D inversion recovery late gadolinium enhancement (LGE) images were acquired to evaluate focal myocardial fibrosis. Finally, 15-min post-contrast, MOLLI T1 mapping was repeated in a protocol identical to that used for pre-contrast T1 mapping. The presence of LGE was visually assessed.

Pre- and post-contrast MOLLI images were processed using the open-source software MRmap v1.4 [19] under IDL. Images were corrected for respiratory motion when needed. T1 maps were exported to Osirix 5.7 (Pixmeo, Geneva, Switzerland) and pre- and post-myocardial T1 times were measured in six regions-of-interest (ROI) in the myocardium (anterior, anterolateral, inferolateral, inferior, inferoseptal, anteroseptal). We calculated the average T1 time of the six different ROIs. Areas of ischemic focal fibrosis identified by late gadolinium enhancement were excluded from the analysis. The partition coefficient lambda $(\lambda)$ and ECV were then computed according to the formula [20].

End-diastolic and end-systolic LV and RV volumes as well as LV mass and presence of LGE were analyzed using the freely available software Segment 2.0 (Medviso, Lund, Sweden), as previously described. LGE was considered present if myocardial enhancement was observed on both short-axis and long-axis views. The total LGE volume was calculated by summing the LGE volume of all slices, and the ratio (\%) of LGE was then calculated using the same software.

\section{Follow-up}

Patients were prospectively followed by ambulatory visits and telephone calls at 6-month intervals. Clinical and survival status was obtained by follow up visits and by phone contact with the patients, their relatives, and their physician if necessarily. The primary outcome was a composite of all-cause mortality or a first hospitalization for HF. Vital status was ascertained by medical record review. First HF hospitalization was defined as patients treated in the emergency room or admitted to a hospital and requiring intravenous diuretics. Patients had at least one symptom and 2 signs of HF (peripheral edema, pulmonary crackles, high NT-proBNP level, radiological signs of pulmonary congestion or hemodynamic evidence).

\section{Statistical analysis}

Statistical analyses were performed using SPSS version 22 (International Business Machines, Inc., Armonk, New
York, USA) and STATA version 11 (Stata Corporation, College Station, Texas, USA) software. All tests were two-sided, and a $P<0.05$ was considered statistically significant. Continuous variables were expressed as mean \pm 1 SD if normally distributed or as medians (25th and 75th percentiles) if not normally distributed. Categorical variables were expressed as counts and percentages. Comparison between groups was performed using ANOVA, chi-square test, or unpaired t-tests when appropriate. We determined an ECV age and sex-adjusted cutoff value corresponding to the upper $95 \%$ confidence interval of 26 age- and sex matched volunteers (ECV $\geq 33 \%$ ).

Logistic regression was performed to determine predictors of abnormal diffuse fibrosis (ECV above or below 95\% confidence intervals in controls). For this purpose, after univariate comparison of the two groups, parameters with a $P<0.10$ were proposed for inclusion in the multiple logistic regression analysis with a backward selection procedure.

Event-free survival was estimated using Kaplan-Meier methods and Cox regression analysis. All baseline and imaging variables were initially proposed for inclusion in a univariate Cox proportional hazard model. To avoid colinearity in the Cox regression model, the correlation coefficients between covariates were examined. In cases of colinearity $(r>0.50)$, only the strongest of the two covariates was proposed for inclusion into the multivariate model. After univariate Cox regression analysis, all significant variables $(P<0.10)$ were entered into a stepwise forward multivariate Cox regression model. Two different models were evaluated using ECV as either a continuous or a categorical variable (ECV $\geq 33 \%$ ).

A prognostic score was established in patients followed up for at least 6 months. The accuracy of ECV, risk score or LGE to predict composite outcome were evaluated by area under the receiver operator curve (ROC) curves. This score was validated in 53 consecutive HFpEF patients recruited between October 2016 and July 2017.

\section{Results \\ Baseline characteristics}

Between December 2014 and October 2016, a total of 118 consecutive HFpEF patients $(78 \pm 8$ years, $63 \%$ women) and 26 age- and sex-matched controls (76 \pm 5 years, $62 \%$ women) were included in the study. The demographic, clinical, and laboratory characteristics of HFpEF patients and controls are summarized in Table 1. We observed a high prevalence of established cardiovascular risk factors in our HFpEF population; including arterial hypertension (93\%), diabetes (39\%), hypercholesterolemia (67\%), and higher body mass index (BMI). HFpEF patients had worse renal 
Table 1 Baseline characteristics of HFpEF patients and age- and sex-matched controls

\begin{tabular}{|c|c|c|c|}
\hline & HFpEF $(n=118)$ & Healthy Controls $(n=26)$ & $P$ \\
\hline Age (years) & $78 \pm 8$ & $76 \pm 5$ & 0.28 \\
\hline Body mass index $\left(\mathrm{kg} / \mathrm{m}^{2}\right)$ & $29 \pm 7$ & $25 \pm 4$ & 0.011 \\
\hline Female $(n, \%)$ & $74(63)$ & $16(62)$ & 0.91 \\
\hline Heart rate (bpm) & $73 \pm 14$ & $67 \pm 9$ & 0.040 \\
\hline Systolic blood pressure $(\mathrm{mmHg})$ & $136 \pm 21$ & $144 \pm 22$ & 0.069 \\
\hline Diastolic blood pressure (mmHg) & $75 \pm 13$ & $82 \pm 12$ & 0.014 \\
\hline NYHA functional class III-IV (n, \%) & $53(45)$ & $0(0)$ & $<0.001$ \\
\hline \multicolumn{4}{|l|}{ Medical history } \\
\hline Atrial fibrillation (n, \%) & $73(62)$ & $0(0)$ & $<0.001$ \\
\hline Ischemic cardiomyopathy (n, \%) & $39(33)$ & $0(0)$ & $<0.001$ \\
\hline Previous valvular surgery (n, \%) & $12(10)$ & $0(0)$ & 0.12 \\
\hline Previous heart failure episode & $84(71)$ & $0(0)$ & $<0.001$ \\
\hline \multicolumn{4}{|l|}{ Cardiovascular risk factors } \\
\hline Smoking (n, \%) & $47(40)$ & $6(23)$ & 0.10 \\
\hline Hypertension (n, \%) & $109(93)$ & $16(62)$ & $<0.001$ \\
\hline Diabetes (n, \%) & $46(39)$ & $1(4)$ & $<0.001$ \\
\hline Family history of cardiovascular disease (n, \%) & $24(21)$ & $3(12)$ & 0.40 \\
\hline Hypercholesterolemia (n, \%) & $78(67)$ & $23(88)$ & 0.027 \\
\hline \multicolumn{4}{|l|}{ Medication } \\
\hline Diuretics other than MRA $(n, \%)$ & $94(80)$ & $2(8)$ & $<0.001$ \\
\hline MRA $(n, \%)$ & $23(19)$ & $0(0)$ & 0.01 \\
\hline Beta-blockers (n, \%) & $76(64)$ & $3(12)$ & $<0.001$ \\
\hline ACE-I or ARB (n, \%) & $76(64)$ & $9(35)$ & 0.005 \\
\hline Statins $(n, \%)$ & $54(46)$ & $5(19)$ & 0.01 \\
\hline \multicolumn{4}{|l|}{ Laboratory characteristics } \\
\hline eGFR $\left(\mathrm{ml} / \mathrm{min} / 1.73 \mathrm{~m}^{2}\right)$ & $59 \pm 23$ & $70 \pm 18$ & 0.018 \\
\hline Hemoglobin (g/dl) & $11.8 \pm 1.9$ & $14.0 \pm 1.3$ & $<0.001$ \\
\hline NT-proBNP (pg/ml) & $1747[374 ; 34,306] £$ & $111[29 ; 393] £$ & 0.001 \\
\hline
\end{tabular}

£ Median [min; max]

$A C E-l$ angiotensin converting enzyme inhibitor, $A R B$ angiotensin receptor blocker, eGFR estimated glomerular filtration rate; MRA:

function and lower hemoglobin and hematocrit levels than healthy controls, and $62 \%$ of patients had a history of atrial fibrillation.

Table 2 summarizes echocardiographic and CMR measurements. As expected, compared to age- and sex-matched healthy controls, HFpEF patients had higher E/e' ratio, higher indexed LA and RA volumes, higher RV/RA gradient, and worse RV function as evaluated by TAPSE and FAC. Prevalence of pulmonary hypertension (RV-RA gradient $>35 \mathrm{mmHg}$ ) in our population was $39 \%(n=46)$. By CMR, HFpEF patients had a slightly lower LVEF and higher indexed LV mass than age- and sex-matched healthy controls. Twenty-six HFpEF patients had LGE $(8=$ focal spots, $18=$ ischemic pattern). When present, the average percentage of LGE was $5.5 \pm 2.9 \%$.
ECV was significantly higher in HFpEF patients than in the healthy control group (Fig. 1). Forty-nine (42\%) HFpEF patients had significant diffuse fibrosis based on the ECV cutoff (defined as $\geq 33 \%$ ). Sixteen (14\%) HFpEF patients had impaired RV systolic function defined as RVEF $\leq 45 \%$ by CMR. HFpEF patients with RV dysfunction had higher ECV ( $36 \pm 6 \%$ vs. 32 $\pm 4 \%, P<0.001)$, higher indexed LA volume $(81 \pm$ $26 \mathrm{ml} / \mathrm{m}^{2}$ vs. $\left.64 \pm 28 \mathrm{ml} / \mathrm{m}^{2}, P=0.03\right)$, lower LVEF $(59 \pm 7 \%$ vs. $64 \pm 8 \%, P=0.010)$, lower TAPSE $(14 \pm$ $5 \mathrm{~mm}$ vs. $19 \pm 5 \mathrm{~mm}, P<0.001)$, lower FAC $(32 \pm 6 \%$ vs. $43 \pm 8 \%, P<0.001)$, and higher septal $\mathrm{E} / \mathrm{e}$ ' ratio ( $24 \pm 9$ vs. $17 \pm 7, P=0.001$ ).

Diabetic HFpEF patients were younger ( $75 \pm 9$ vs. $80 \pm$ 7 years, $P=0.002$ ), had a higher body mass index (BMI) (31.2 \pm 6.9 vs. $27.3 \pm 6.1, P=0.002$ ), a lower atrial fibrillation 
Table 2 Echocardiographic and CMR parameters of HFpEF patients and age- and sex-matched controls

\begin{tabular}{|c|c|c|c|}
\hline & HFpEF $(n=118)$ & Healthy Controls $(n=26)$ & $P$ \\
\hline \multicolumn{4}{|l|}{ Echocardiography } \\
\hline LV ejection fraction (\%) & $64 \pm 7$ & $64 \pm 5$ & 0.93 \\
\hline Transmitral peak E velocity max $(\mathrm{m} / \mathrm{s})$ & $91 \pm 29$ & $55 \pm 9$ & $<0.001$ \\
\hline Transmitral E deceleration time (ms) & $160 \pm 62$ & $197 \pm 35$ & 0.004 \\
\hline E/e' septal ratio & $18.1 \pm 7.3$ & $9.4 \pm 1.7$ & $<0.001$ \\
\hline RV/RA gradient (mmHg) & $32 \pm 11$ & $19 \pm 5$ & $<0.001$ \\
\hline RA volume index $\left(\mathrm{ml} / \mathrm{m}^{2}\right)$ & $35 \pm 20$ & $18 \pm 5$ & $<0.001$ \\
\hline RV fractional area change (\%) & $41 \pm 9$ & $47 \pm 8$ & 0.008 \\
\hline RV FAC $\leq 35 \%(n, \%)$ & $32(27)$ & $0(0)$ & $<0.001$ \\
\hline TAPSE $(m m)$ & $19 \pm 5$ & $24 \pm 4$ & $<0.001$ \\
\hline TAPSE $\leq 16$ mm (n, \%) & $47(40)$ & $1(4)$ & $<0.001$ \\
\hline \multicolumn{4}{|l|}{ Cardiac MR } \\
\hline LVM index $\left(\mathrm{g} / \mathrm{m}^{2}\right)$ & $68 \pm 15$ & $58 \pm 12$ & 0.003 \\
\hline LV EDV index $\left(\mathrm{ml} / \mathrm{m}^{2}\right)$ & $73 \pm 18$ & $63 \pm 11$ & 0.006 \\
\hline LVEF (\%) & $63 \pm 8$ & $67 \pm 5$ & 0.024 \\
\hline RV EDV index $\left(\mathrm{ml} / \mathrm{m}^{2}\right)$ & $82 \pm 28$ & $67 \pm 11$ & 0.005 \\
\hline RVEF (\%) & $57 \pm 9$ & $60 \pm 6$ & 0.052 \\
\hline RVEF $\leq 45 \%(n, \%)$ & $16(14)$ & $2(8)$ & 0.41 \\
\hline LV mass/volume ratio & $0.96 \pm 0.20$ & $0.94 \pm 0.19$ & 0.71 \\
\hline LA volume index (ml) & $66 \pm 29$ & $32 \pm 10$ & $<0.001$ \\
\hline Myocardium native $\mathrm{T} 1$ time (ms) & $1109 \pm 82$ & $1144 \pm 47$ & 0.038 \\
\hline Myocardium post contrast T1 time (ms) & $353 \pm 56$ & $381 \pm 64$ & 0,028 \\
\hline ECV (\%) & $32.9 \pm 4.8$ & $28.2 \pm 2.4$ & $<0.001$ \\
\hline Lambda coefficient & $0.52 \pm 0.08$ & $0.49 \pm 0.05$ & 0.051 \\
\hline$E C V \geq 33 \%(n, \%)$ & $49(42)$ & $0(0)$ & $<0.001$ \\
\hline Late gadolinium enhancement (n, \%) & $26(22)$ & $0(0)$ & 0.022 \\
\hline
\end{tabular}

Values are mean $\pm \mathrm{SD}$. $L A$ left atrium, $L V$ left ventricle, $R V$ right ventricle, $R A$ right atrium, $B M I$ body mass index

TAPSE tricuspid annular plane systolic excursion, $L V M$ left ventricular mass, EF ejection fraction, EDV end-diastolic volume, ECV extracellular volume

history rate $(48 \%$ vs. $71 \%, P=0.012)$, and more known ischemic cardiomyopathy (43\% vs. $26 \%, P=0.055)$ than non-diabetic HFpEF patients. Echocardiographic and CMR parameters were quite similar except for a trend to higher indexed LV mass in diabetics $(71.0 \pm 12.6$ vs. $65.6 \pm 16.1 \mathrm{~g} /$ $\left.\mathrm{m}^{2}, P=0.056\right)$. Diabetic HFpEF patients presented more frequently $\mathrm{ECV} \geq 33 \%$ than non-diabetic patients ( $52 \%$ vs. $35 \%, P=0.061$ ).

\section{Predictors of high ECV}

Table 3 compares HFpEF patients with high and low $\mathrm{ECV}$ values. ECV and the lambda coefficient were different between the two groups, demonstrating that the difference in ECV was not solely the result of a significant difference in hematocrit level..

HFpEF patients with ECV $\geq 33 \%$ had lower BMI, lower hematocrit, higher prevalence of diabetes, higher transmitral peak $\mathrm{E}$ wave velocity and higher $\mathrm{E} / \mathrm{e}$ ' ratio. A higher proportion of HFpEF patients with $\mathrm{ECV} \geq 33 \%$ had an impaired RV systolic function (CMR RVEF $\leq 45 \%$ ( $20 \%$ vs. $9 \%, P=0.073)$. There were no differences in NT-proBNP and LGE in HFpEF patients between the two groups.

In multivariate logistic regression, $\mathrm{BMI}(\mathrm{OR}=0.92$ [0.86-0.98], $P=0.011)$, presence of diabetes $(\mathrm{OR}=2.62$ [1.11-6.18], $P=0.028)$, and higher transmitral peak $\mathrm{E}$ wave velocity $(\mathrm{OR}=1.02[1.00-1.03], P=0.022)$ were significantly associated with high ECV value.

\section{Outcomes}

During a mean follow-up of $11 \pm 6$ months, we observed 43 events (11 deaths and 32 hospitalizations for HF) (Fig. 2a). The primary outcome (all-cause mortality or first hospitalization for HF) occurred in 38 patients (32\%). Only one patient was lost to follow up. The percentage of combined event at 18 months in our population was $50 \%$. 


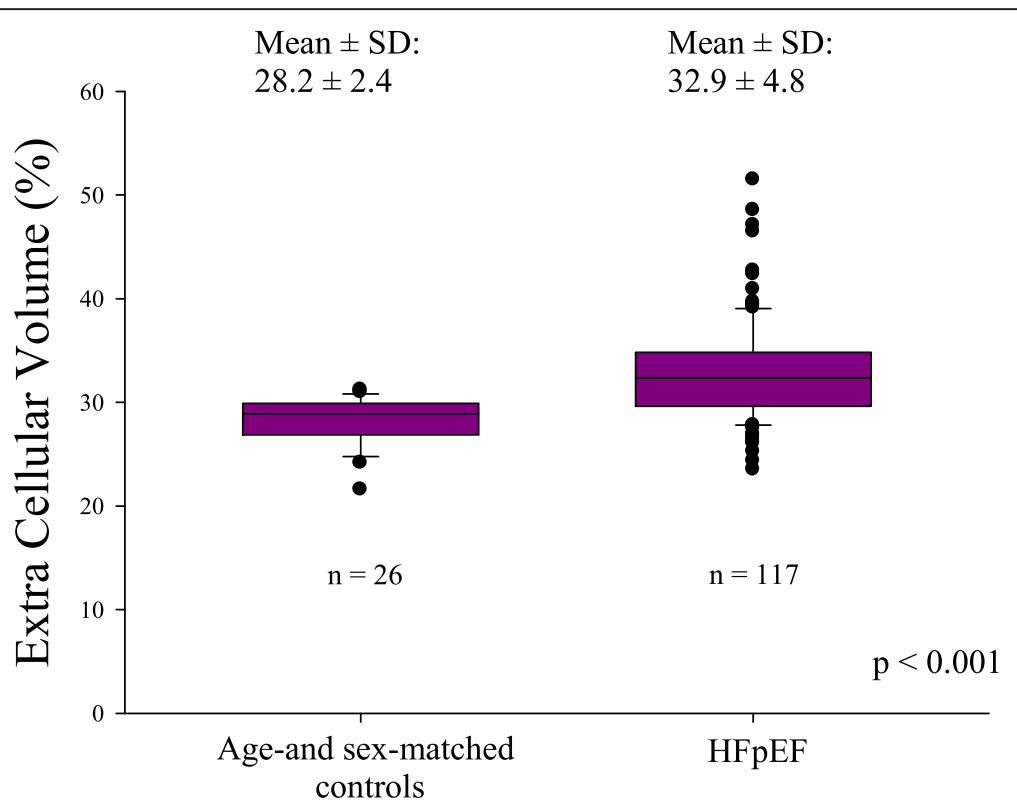

Fig. 1 Extracellular volume fraction (ECV) values between age- and sex-matched healthy controls and heart failure with preserved ejection fraction (HFpEF) patients

In univariate Cox regression analysis, lower eGFR, lower hemoglobin, presence of diabetes, higher transmitral $\mathrm{E}$ wave velocity, and $\mathrm{ECV} \geq 33 \%$ were significant predictors of the composite outcome (Table 4). In multivariate Cox regression analysis, only the presence of diabetes $(\mathrm{HR}=1.98[1.04 ; 3.76], P=0.038)$ and hemoglobin level ( $\mathrm{HR}=0.81[0.67 ; 0.98], P=0.028)$ were significantly associated with the composite outcome (Table 5). The ability of either ECV as a continuous variable or ECV as a categorical variable (ECV > 33\%) to improve this model was then tested and added significant prognostic information $\left(x^{2} 4.33\right.$, $P=0.037$ and $X^{2} 4.46, P=0.035$, respectively), as opposed to transmitral peak $\mathrm{E}$ wave velocity and eGFR ( $x^{2} 3.00, p=0.083$ and $x^{2} 2.86, p=0.091$ respectively). The ability of the model to discriminate outcome was good with an area under the curve (AUC) of 0.76 . Kaplan-Meier event-free survival curves showed that HFpEF patients with ECV $\geq 33 \%$ had poorer one-year prognosis than those with $\mathrm{ECV}<33 \%(56 \pm 8 \%$ vs 82 $\pm 5 \%, P=0.001$, Fig. 2b).

A prognostic score was established based on the significant predictors of composite outcome in patients with at least 6 months of follow up $(n=97)$. Diabetes, hemoglobin, and ECV > 33\% were thus used to build the risk score. ROC curves showed a better discrimination with the prognostic score and ECV (c-statistic of 0,76 and 0,67 respectively) compared to LGE (c statistic 0,51) (Additional file 1). In the validation cohort of 53 patients, 2 deaths and 9 HF hospitalizations were observed during a mean follow up of $11 \pm 5$ months.
Our risk score based on the initial cohort and applied in the validation cohort had an AUC of 0.71 to predict primary outcome.

\section{Discussion}

We sought to evaluate the presence, associations, and prognostic significance of quantification of ECV using $3 \mathrm{~T}$ CMR in a prospective and well-characterized HFpEF cohort. The salient findings of our study are as follows. Mean ECV by T1-mapping was significantly higher in HFpEF patients than in age-matched healthy controls; ECV in HFpEF patients was related to the presence of anemia and diabetes and associated with altered diastolic function by echocardiography and lower CMR RV systolic function; and finally, increased ECV and the presence of anemia and diabetes were independent risk markers of short-term poor prognosis with increased rehospitalization or all-cause mortality in HFpEF.

\section{HFpEF}

HFpEF is a heterogeneous disease observed mainly in the aging population. We and others demonstrated that aging is associated with structural changes in the heart, such as alterations of diastolic function, increased stiffening, LV and atrial remodeling characterized by decreased LV and RV volumes and mass, increased atrial volumes, and an increase in ECV [21]. It is crucial to separate age-related changes from disease-related processes associated with HFpEF. Therefore, we compared our population to carefully 
Table 3 Baseline characteristics of HFpEF patients with ECV $<$ or $\geq 33 \%$

\begin{tabular}{|c|c|c|c|}
\hline & HFpEF with ECV <33\% $(n=68)$ & HFpEF with ECV $\geq 33 \%(n=49)$ & $P$ \\
\hline \multicolumn{4}{|l|}{ Baseline characteristics } \\
\hline Age (years) & $78 \pm 8$ & $79 \pm 9$ & 0.49 \\
\hline Body mass index $\left(\mathrm{kg} / \mathrm{m}^{2}\right)$ & $30 \pm 7$ & $28 \pm 7$ & 0.082 \\
\hline Female $(n, \%)$ & $41(60)$ & $33(67)$ & 0.44 \\
\hline Systolic blood pressure (mmHg) & $137 \pm 21$ & $135 \pm 21$ & 0.54 \\
\hline Diastolic blood pressure ( $\mathrm{mmHg}$ ) & $76 \pm 12$ & $74 \pm 14$ & 0.45 \\
\hline NYHA functional class III-IV (n, \%) & $28(41)$ & $24(49)$ & 0.57 \\
\hline \multicolumn{4}{|l|}{ Laboratory characteristics } \\
\hline eGFR $\left(\mathrm{ml} / \mathrm{min} / 1.73 \mathrm{~m}^{2}\right)$ & $58 \pm 20$ & $60 \pm 28$ & 0.56 \\
\hline Hemoglobin (g/dl) & $12.1 \pm 1.8$ & $11.3 \pm 2.0$ & 0.020 \\
\hline NT-proBNP (pg/ml) & $1584[432 ; 34,306] £$ & $1889[374 ; 27,736] £$ & 0.40 \\
\hline \multicolumn{4}{|l|}{ Medical history } \\
\hline Atrial fibrillation (n, \%) & $42(62)$ & $30(61)$ & 0.90 \\
\hline Ischemic cardiomyopathy (n, \%) & $23(34)$ & $16(33)$ & 0.94 \\
\hline Previous valvular surgery $(\mathrm{n}, \%)$ & $8(12)$ & $4(8)$ & 0.76 \\
\hline \multicolumn{4}{|l|}{ Cardiovascular risk factors } \\
\hline Smoking (n, \%) & $29(43)$ & $18(37)$ & 0.52 \\
\hline Hypertension (n, \%) & $64(94)$ & $44(92)$ & 0.37 \\
\hline Diabetes (n, \%) & $22(33)$ & $24(49)$ & 0.069 \\
\hline Family history of cardiovascular disease $(n, \%)$ & $14(21)$ & $10(20)$ & 0.98 \\
\hline Hypercholesterolemia (n, \%) & $47(70)$ & $30(61)$ & 0.29 \\
\hline \multicolumn{4}{|l|}{ Echocardiography } \\
\hline Left ventricular ejection fraction (\%) & $64 \pm 7$ & $63 \pm 8$ & 0.66 \\
\hline Transmitral peak E velocity max (m/s) & $86 \pm 29$ & $98 \pm 26$ & 0.025 \\
\hline Transmitral E deceleration time (ms) & $155 \pm 62$ & $167 \pm 63$ & 0.35 \\
\hline E/e' septal ratio & $16 \pm 6$ & $20 \pm 8$ & 0.004 \\
\hline RV/RA gradient (mmHg) & $31 \pm 11$ & $34 \pm 11$ & 0.13 \\
\hline RA volume index $\left(\mathrm{ml} / \mathrm{m}^{2}\right)$ & $34 \pm 19$ & $36 \pm 22$ & 0.75 \\
\hline RV fractional area change (\%) & $42 \pm 8$ & $42 \pm 11$ & 0.84 \\
\hline TAPSE (mm) & $19 \pm 5$ & $18 \pm 5$ & 0.11 \\
\hline \multicolumn{4}{|l|}{ Cardiac MR } \\
\hline LVM index $\left(\mathrm{g} / \mathrm{m}^{2}\right)$ & $67 \pm 14$ & $66 \pm 16$ & 0.81 \\
\hline LV EDV index $\left(\mathrm{ml} / \mathrm{m}^{2}\right)$ & $71 \pm 16$ & $72 \pm 20$ & 0.58 \\
\hline LVEF (\%) & $63 \pm 8$ & $63 \pm 8$ & 0.997 \\
\hline RV EDV index $\left(\mathrm{ml} / \mathrm{m}^{2}\right)$ & $77 \pm 22$ & $86 \pm 32$ & 0.097 \\
\hline RVEF (\%) & $56 \pm 7$ & $56 \pm 10$ & 0.98 \\
\hline RVEF $\leq 45 \%(n, \%)$ & $6(9)$ & $10(20)$ & 0.073 \\
\hline LV mass/volume ratio & $0.97 \pm 0.21$ & $0.94 \pm 0.19$ & 0.43 \\
\hline LA volume index $\left(\mathrm{ml} / \mathrm{m}^{2}\right)$ & $65 \pm 29$ & $68 \pm 28$ & 0.68 \\
\hline Myocardium native $\mathrm{T} 1$ time (ms) & $1109 \pm 83$ & $1107 \pm 82$ & 0.88 \\
\hline Myocardium post contrast T1 time (ms) & $362 \pm 58$ & $340 \pm 52$ & 0.036 \\
\hline ECV (\%) & $30.0 \pm 2.2$ & $37.0 \pm 4.3$ & $<0.001$ \\
\hline Lambda coefficient & $0.48 \pm 0.05$ & $0.57 \pm 0.08$ & $<0.001$ \\
\hline Late gadolinium enhancement & $13(19)$ & $13(28)$ & 0.29 \\
\hline
\end{tabular}

Values are mean \pm SD. $:$ median (min, max); $L A$ left atrium, $L V$ left ventricle, $R V$ right ventricle, $R A$ right atrium, $B M I$ body mass index, TAPSE tricuspid annular plane systolic excursion, LVM left ventricular mass, EF ejection fraction, EDV end-diastolic volume, ECV extracellular volume 
age- and sex-matched healthy subjects, allowing for a better understanding of these two processes. As compared to previous work [22, 23], our HFpEF population was older and had more comorbidities, probably reflecting a less selected population but a more advanced stage because patients were highly symptomatic with high NT-proBNP levels (45\% NYHA III/IV). Overall, and in accordance with prior reports, we observed relatively poor outcomes with a high rehospitalization rate and high mortality despite optimal medical treatment.

\section{Role of extracellular matrix abnormalities in $\mathrm{HFpEF}$}

Extracellular matrix abnormalities causing LV stiffening and secondary LV diastolic dysfunction are probably among the main potential pathophysiologic mechanisms involved in HFpEF. Previous studies using endomyocardial biopsies or autopsies have already shown that the extent of fibrosis is higher in HFpEF patients than in controls [7].Studies have demonstrated that ECV closely correlates with histologically determined diffuse interstitial fibrosis, providing a non-invasive estimation for its quantification; however, only a few studies have a

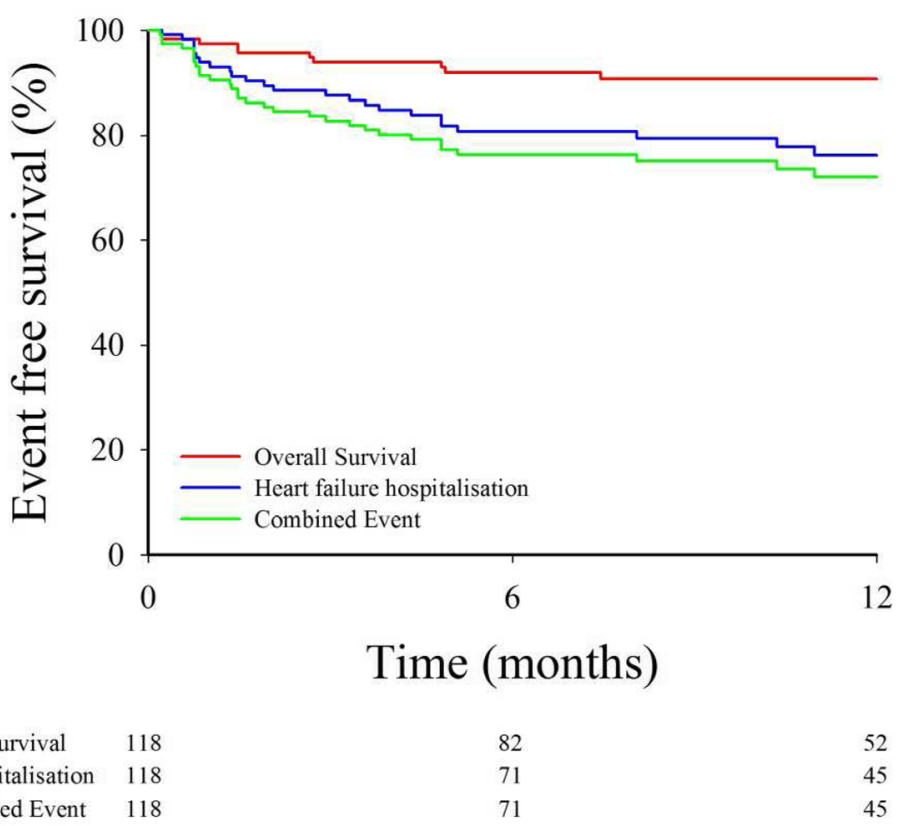

b

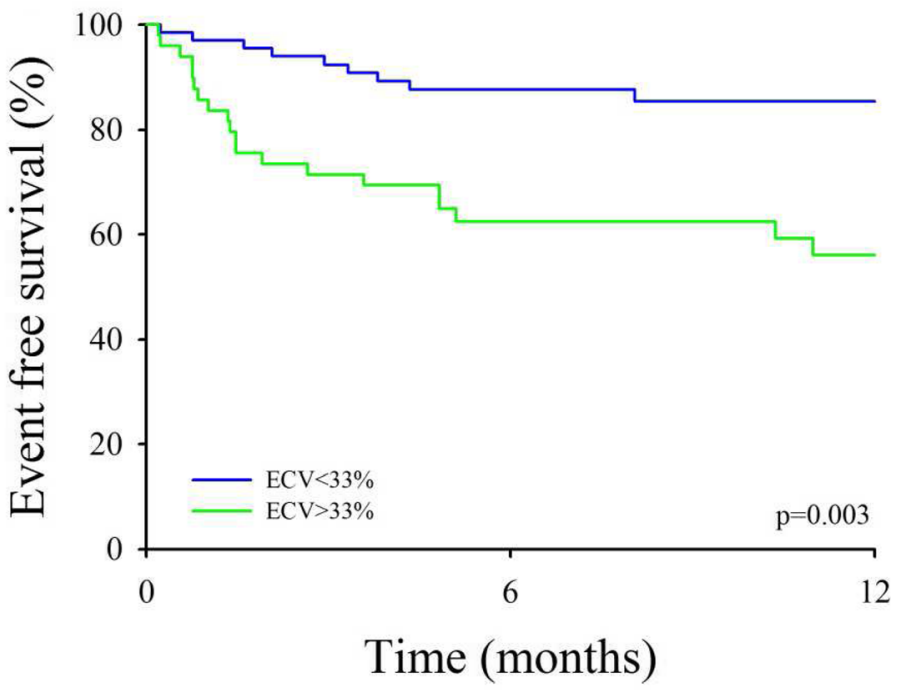

$\begin{array}{llll}\mathrm{ECV}<33 \% & 68 & 47 & 28\end{array}$

Fig. 2 (a) Mortality and HF hospitalization Kaplan Meier Curves (b) Event-free survival in HFpEF patients according to ECV cutoff 
Table 4 Univariate Cox analysis for composite event (death and first HF)

\begin{tabular}{|c|c|c|}
\hline & \multicolumn{2}{|c|}{ Composite outcome (death or first HF) } \\
\hline & HR [95\%lC] & $P$ \\
\hline Age (years) & $1.00[0.96 ; 1.04]$ & 0.96 \\
\hline Body surface area $\left(\mathrm{m}^{2}\right)$ & $0.29[0.08 ; 1.08]$ & 0.065 \\
\hline Female (n, \%) & $1.63[0.79 ; 3.36]$ & 0.19 \\
\hline Heart rate (bpm) & $1.02[1.00 ; 1.04]$ & 0.086 \\
\hline Systolic blood pressure (mmHg) & $0.99[0.97 ; 1.01]$ & 0.18 \\
\hline Diastolic blood pressure (mmHg) & $0.97[0.95 ; 1.00]$ & 0.064 \\
\hline NYHA functional class III-IV & $1.18[0.62 ; 2.26]$ & 0.62 \\
\hline \multicolumn{3}{|l|}{ Laboratory characteristics } \\
\hline $\operatorname{eGFR}\left(\mathrm{ml} / \mathrm{min} / 1.73 \mathrm{~m}^{2}\right)$ & $0.98[0.96 ; 0.99]$ & 0.040 \\
\hline Hemoglobin (g/dl) & $0.80[0.66 ; 0.96]$ & 0.020 \\
\hline NT-proBNP (pg/ml) & $1.12[0.82 ; 1.55]$ & 0.48 \\
\hline \multicolumn{3}{|l|}{ Medical history } \\
\hline Atrial fibrillation (n, \%) & $1.15[0.58 ; 2.25]$ & 0.69 \\
\hline Ischemic cardiomyopathy (n, \%) & $1.03[0.52 ; 2.04]$ & 0.94 \\
\hline Previous valvular surgery $(\mathrm{n}, \%)$ & $1.16[0.35 ; 3.83]$ & 0.81 \\
\hline Previous heart failure episode & $1.17[0.50 ; 2.73]$ & 0.72 \\
\hline \multicolumn{3}{|l|}{ Cardiovascular risk factors } \\
\hline Smoking (n, \%) & $0.99[0.51 ; 1.92]$ & 0.98 \\
\hline Hypertension (n, \%) & $0.81[0.25 ; 2.68]$ & 0.73 \\
\hline Diabetes (n, \%) & $2.15[1.13 ; 4.08]$ & 0.020 \\
\hline Family history of cardiovascular disease $(n, \%)$ & $0.97[0.45 ; 2.12]$ & 0.94 \\
\hline Hypercholesterolemia (n, \%) & $1.04[0.51 ; 2.11]$ & 0.91 \\
\hline \multicolumn{3}{|l|}{ Echocardiography } \\
\hline LV ejection fraction (\%) & $1.03[0.98 ; 1.08]$ & 0.20 \\
\hline Transmitral peak E velocity max (m/s) & $1.01[1.00 ; 1.02]$ & 0.025 \\
\hline E/e' septal ratio & $1.02[0.98 ; 1.06]$ & 0.42 \\
\hline RV/RA gradient (mmHg) & $1.01[0.98 ; 1.04]$ & 0.60 \\
\hline RA volume index $\left(\mathrm{ml} / \mathrm{m}^{2}\right)$ & $1.00[0.99 ; 1.02]$ & 0.53 \\
\hline RV fractional area change (\%) & $2.01[0.06 ; 73.25]$ & 0.70 \\
\hline TAPSE (mm) & $0.94[0.89 ; 1.00]$ & 0.064 \\
\hline \multicolumn{3}{|l|}{ Cardiac MR } \\
\hline LVM index $\left(\mathrm{g} / \mathrm{m}^{2}\right)$ & $0.99[0.98 ; 1.01]$ & 0.62 \\
\hline LV EDV index $\left(\mathrm{ml} / \mathrm{m}^{2}\right)$ & $0.99[0.98 ; 1.01]$ & 0.58 \\
\hline LVEF (\%) & $1.03[0.99 ; 1.07]$ & 0.19 \\
\hline RV EDV index $\left(\mathrm{ml} / \mathrm{m}^{2}\right)$ & $1.00[0.99 ; 1.01]$ & 0.59 \\
\hline RVEF (\%) & $0.99[0.96 ; 1.04]$ & 0.81 \\
\hline LV mass/volume ratio & $1.24[0.06 ; 25.3]$ & 0.89 \\
\hline LA volume index $\left(\mathrm{ml} / \mathrm{m}^{2}\right)$ & $1.00[0.98 ; 1.02]$ & 0.87 \\
\hline Myocardium native $\mathrm{T} 1$ (ms) & $1.01[0.99 ; 1.01]$ & 0.23 \\
\hline ECV (\%) & $1.07[1.01 ; 1.12]$ & 0.015 \\
\hline$E C V \geq 33 \%$ & $2.62[1.35 ; 5.09]$ & 0.005 \\
\hline LGE (\%) & $1.08[0.96 ; 1.21]$ & 0.22 \\
\hline
\end{tabular}


Table 5 Multivariate Cox analysis for event-free survival in HFpEF

\begin{tabular}{|c|c|c|c|c|c|}
\hline & $\mathrm{HR}([95 \% \mathrm{Cl}]$ & $P$ & $X^{2}$ to remove & $X^{2}$ to enter & $P$ \\
\hline Diabetes mellitus & $1.98[1.04 ; 3.76]$ & 0.038 & 4.327 & & \\
\hline Hemoglobin & $0.81[0.67 ; 0.98]$ & 0.028 & 4.961 & & \\
\hline Mean E wave & & & & 3.00 & 0.083 \\
\hline eGFR & & & & 2.86 & 0.091 \\
\hline Model 1: ECV (continuous variable) & $1.07[1.00 ; 1.13]$ & & & 4.33 & 0.037 \\
\hline Model 2: ECV 33\% & $2.00[1.00 ; 4.03]$ & & & 4.46 & 0.035 \\
\hline
\end{tabular}

$\mathrm{Cl}$ confidence interval

evaluated ECV in HFpEF patients [15, 22, 23]. Our findings confirm that high ECV likely reflecting diffuse extracellular matrix abnormalities, a potential surrogate for myocardial fibrosis, may play a key role in the pathophysiology of HFpEF. Indeed, ECV was significantly increased relative to the age-matched population, yet only $42 \%$ of our HFpEF patients had high ECV, suggesting that other mechanisms are involved in HFpEF pathophysiology. Assessment of ECV allows for a direct evaluation of the extracellular space, reflecting interstitial disease. However, it lacks the ability to provide information about the relative contribution of edema, fibrosis, inflammation, or deposition of other extracellular proteins such as amyloid [13] and therefore may not allow for a full understanding of the pathophysiological mechanisms underlying HFpEF. In our study, low BMI, diabetes, and high transmitral peak $\mathrm{E}$ wave velocity were significant determinants of high ECV. High transmitral peak $\mathrm{E}$ wave velocity is a good surrogate for high filling pressures, and it could be easily anticipated as an important determinant of fibrosis.

Surprisingly, we observed that a higher BMI was associated with lower ECV values, suggesting that diffuse fibrosis is not the sole mechanism involved in HFpEF pathogenesis in the obese population. Indeed, adiposity-induced inflammation has considerable adverse effects, including endothelial dysfunction, capillary rarefaction, and mitochondrial dysfunction in both the cardiac and systemic beds [24].

Diabetic cardiomyopathy could be considered as a form of HFpEF, explaining the increased incidence of HF in the diabetic population [25]. This cardiomyopathy is characterized by insulin resistance and a loss of metabolic flexibility, and subsequently by cardiomyocyte hypertrophy and increased fibrosis. Increased reactive oxygen species production is one of the major pathophysiological mechanisms triggered by hyperglycemia and high free fatty acid level.

Finally, native T1 time was surprisingly significantly lower in HFpEF patients than in healthy controls. We can only speculate on the explanation. Possible explanation could be that HFpEF patients have slightly higher intramyocadial iron concentration or more intramyocardial fat, particularly related to presence of diabetes.

\section{Prognosis in HFpEF}

As demonstrated in our study, the prognosis of HFpEF patients is still quite poor. Many studies have compared HFpEF and HFrEF prognosis and demonstrated the same or even a worse mortality and morbidity rate $[26,27]$ for HFpEF than for HFrEF. Our study identified high ECV, hemoglobin and diabetes as independent prognostic predictors for the short-term composite outcome. This result is in accordance with observations by Redfield et al. [28], who showed that profibrotic pathways may contribute to adverse outcomes in diabetic HFpEF patients. However, in our work, presence of LGE was not a significant predictor of the composite outcome $(\mathrm{HR}=1.08[0.96 ; 1.21], p=0.22)$ in univariate analysis, in contrast to another recent study [16]. A potential explanation was that patients in this pre cited study had significantly higher percentage of LGE quantification (13 $\pm 8 \%)$, as opposed to our population where only 26 patients had small amounts of LGE $(5.5 \pm 2.9 \%)$.

The prognostic role of anemia has already been demonstrated in large HFpEF studies (SENIOR [29], MAGGIC [30], ARIC [26], respectively).

\section{Clinical implications}

Because HFpEF syndrome is a heterogeneous disease, better characterization of HFpEF phenotypes based on clinical presentation and biological and/or imaging data is crucial for better designing therapies [31]. In particular, the identification of anemia is relevant because its association with composite outcome could suggest a potential beneficial effect of iron-replacement therapy in HFpEF patients, and this hypothesis could be evaluated in larger randomized trials. Our study contributes to a better understanding of the heterogeneous and complicated nature of HFpEF [32]. Larger studies are needed to confirm our findings and prospectively validate our risk markers in other populations.

\section{Study limitations}

Our study is a single-center study of relatively small size, and its power is limited by a modest number of events, 
which bears a risk of overfitting in multivariable models. Yet our study was prospective, and the echocardiographic and CMR imaging, as well as biomarker sampling, were standardized. Because we excluded patients with contraindications to CMR, in particular renal failure, our conclusions cannot be generalized to all patients with HFpEF. Although we validated ECV assessment by T1 mapping in other populations against histopathology [15] with reasonably good correlation, in this fragile elderly population, we did not sample cardiac biopsies and thus could not ascertain the pathophysiological correlates of increased ECV in our patients. Another limitation is the lack of others SAX slices for the MOLLI acquisition to have a better idea of ECV value in the rest of the myocardium.

Focal fibrosis is probably also very important in HFpEF patients but only 26 patients had LGE.

Moreover, we interpreted CMR data from patients in atrial fibrillation but the impact of atrial fibrillation on MOLLI sequences has not been fully studied yet.

In addition, our study was performed mainly in a white population, and findings might differ in other groups, particularly in African American or other populations. Finally, the presence of different comorbidities is an important confounding factor in HFpEF. Thus, findings might be affected by selection criteria and presence of comorbidities in such HFpEF populations.

\section{Conclusions}

Among HFpEF patients, high ECV likely reflecting increased myocardial fibrosis was associated with BMI, diabetes, and transmitral peak $\mathrm{E}$ wave velocity. Among HFpEF patients, abnormal diffuse myocardial fibrosis estimated by ECV was associated with a higher rate of all-cause death and first HF hospitalization in the short term follow up.

\section{Additional file}

Additional file 1: Risk score. (DOCX 62 kb)

\section{Abbreviations}

2D: Two dimensional; AUC: Area under the curve; BMI: Body mass index; bSSFP: Balanced steady state free precession; CMR: Cardiovascular magnetic resonance; ECG: Electrocardiogram; ECV: Extracellular volume fraction; EDV: End-diastolic volume; EF: Ejection fraction; eGFR: Estimated glomerular filtration rate; ESV: End-systolic volume; FAC: Fractional area change; HF: Heart failure; HFpEF: Heart failure with preserved ejection fraction; HFrEF: Heart failure with reduced ejection fraction; LA: Left atrium/left atrial; LV: Left ventricle/left ventricular; LVM: Left ventricular mass; MOLLI: Modified Look-Locker inversion recovery; NYHA: New York Heart Association; RA: Right atrium/right atrial; ROC: Receiver operator curve; ROI: Region-of-interest; RV: Right ventricle/right ventricular; SAX: Short axis; TAPSE: Tricuspid annular plane systolic excursion

\section{Funding}

This work was funded by a grant of the Fondation Nationale de la Recherche Scientifique of the Belgian Government (FRMS CDR 23597851). ACP is sponsored by a Post-doctorate Clinical Master Specialist of the
Fondation Nationale de la Recherche Scientifique of the Belgian Government (FRSM: SPD 10844948). Dr. Roy is supported by Fondation Damman and Fondation Saint Luc for her fellowship. This work was funded by an unrestricted grant from Astra Zeneca.

\section{Availability of data and materials}

The datasets used and/or analysed during the current study are available from the corresponding author on reasonable request.

\section{Authors' contributions}

CR conceived the study, recruited patients, acquired and analysed CMR data and drafted the manuscript. AS and CDM acquired CMR data and performed statistical analysis. MA, BG and ACP participated in study coordination and acquired and analysed MR studies. JLVO, AP, DVC, CB and ACP participated in design and coordination of the study. ACP conceived the study, participated in its design and coordination and helped to draft the manuscript. All authors read and approved the final manuscript.

Ethics approval and consent to participate

The study was approved by the IRB of the Cliniques St. Luc, Université Catholique de Louvain and all participants gave written permission to participating in the study.

\section{Consent for publication}

NA

\section{Competing interests}

The Cliniques St. Luc UCL has a master clinical research agreement with Philips Medical Instruments, and the MOLLI patch was supplied by Philips Healthcare under the terms of this agreement. The authors declare that they have no competing interests.

\section{Publisher's Note}

Springer Nature remains neutral with regard to jurisdictional claims in published maps and institutional affiliations.

Received: 30 August 2017 Accepted: 19 July 2018

Published online: 08 August 2018

\section{References}

1. Kanwar M, Walter C, Clarke M, Patarroyo-Aponte M. Targeting heart failure with preserved ejection fraction: current status and future prospects. Vasc Health Risk Manag. 2016;12:129-41.

2. Gerber Y, Weston SA, Redfield MM, Chamberlain AM, Manemann SM, Jiang R, Killian JM, Roger VL. A contemporary appraisal of the heart failure epidemic in Olmsted County, Minnesota, 2000 to 2010. JAMA Intern Med. 2015;175:996-1004.

3. Steinberg BA, Zhao $X$, Heidenreich PA, Peterson ED, Bhatt DL, Cannon CP, Hernandez AF, Fonarow GC. Trends in patients hospitalized with heart failure and preserved left ventricular ejection fraction: prevalence, therapies, and outcomes. Circulation. 2012;126:65-75.

4. Paulus WJ, Tschope C. A novel paradigm for heart failure with preserved ejection fraction: comorbidities drive myocardial dysfunction and remodeling through coronary microvascular endothelial inflammation. J Am Coll Cardiol. 2013;62:263-71.

5. Mohammed SF, Borlaug BA, Roger VL, Mirzoyev SA, Rodeheffer RJ, Chirinos $J A$, Redfield MM. Comorbidity and ventricular and vascular structure and function in heart failure with preserved ejection fraction: a communitybased study. Circ Heart Fail. 2012;5:710-9.

6. Fang JC. Heart failure with preserved ejection fraction: a kidney disorder? Circulation. 2016;134:435-7.

7. Mohammed SF, Hussain S, Mirzoyev SA, Edwards WD, Maleszewski JJ, Redfield MM. Coronary microvascular rarefaction and myocardial fibrosis in heart failure with preserved ejection fraction. Circulation. 2015;131:550-9.

8. Campbell KS, Sorrell VL. Cell- and molecular-level mechanisms contributing to diastolic dysfunction in HFpEF. J Appl Physiol (1985). 2015;119:1228-32.

9. Borlaug BA, Lam CS, Roger VL, Rodeheffer RJ, Redfield MM. Contractility and ventricular systolic stiffening in hypertensive heart disease insights into the pathogenesis of heart failure with preserved ejection fraction. J Am Coll Cardiol. 2009;54:410-8. 
10. van Heerebeek $L$, Paulus WJ. Understanding heart failure with preserved ejection fraction: where are we today? Neth Heart J. 2016;24:227-36.

11. Mohammed SF, Majure DT, Redfield MM. Zooming in on the microvasculature in heart failure with preserved ejection fraction. Circ Heart Fail. 2016;9(7):e003272. https://doi.org/10.1161/CIRCHEARTFAILURE.116. 003272.

12. Flett AS, Hayward MP, Ashworth MT, Hansen MS, Taylor AM, Elliott PM, McGregor C, Moon JC. Equilibrium contrast cardiovascular magnetic resonance for the measurement of diffuse myocardial fibrosis: preliminary validation in humans. Circulation. 2010;122:138-44.

13. Moon JC, Messroghli DR, Kellman P, Piechnik SK, Robson MD, Ugander M, Gatehouse PD, Arai AE, Friedrich MG, Neubauer S, Schulz-Menger J, Schelbert EB. Myocardial T1 mapping and extracellular volume quantification: a Society for Cardiovascular Magnetic Resonance (SCMR) and CMR working Group of the European Society of cardiology consensus statement. J Cardiovasc Magn Reson. 2013;15:92.

14. de Meester de Ravenstein C, Bouzin C, Lazam S, Boulif J, Amzulescu M, Melchior J, Pasquet A, Vancraeynest D, Pouleur AC, Vanoverschelde JL, Gerber BL. Histological validation of measurement of diffuse interstitial myocardial fibrosis by myocardial extravascular volume fraction from modified look-locker imaging (MOLLI) T1 mapping at 3 T. J Cardiovasc Magn Reson. 2015;17:48

15. Duca F, Kammerlander AA, Zotter-Tufaro C, Aschauer S, Schwaiger ML, Marzluf BA, Bonderman D, Mascherbauer J. Interstitial fibrosis, functional status, and outcomes in heart failure with preserved ejection fraction: insights from a prospective cardiac magnetic resonance imaging study. Circ Cardiovasc Imaging. 2016;9

16. Kato S, Saito N, Kirigaya H, Gyotoku D, linuma N, Kusakawa Y, Iguchi K, Nakachi T, Fukui K, Futaki M, Iwasawa T, Taguri M, Kimura K, Umemura S. Prognostic significance of quantitative assessment of focal myocardial fibrosis in patients with heart failure with preserved ejection fraction. Int J Cardiol. 2015;191:314-9.

17. Ponikowski P, Voors AA, Anker SD. 2016 ESC guidelines for the diagnosis and treatment of acute and chronic heart failureThe task force for the diagnosis and treatment of acute and chronic heart failure of the European Society of Cardiology (ESC) developed with the special contribution of the heart failure association (HFA) of the ESC. Eur Heart J. 2016;37:2129-200.

18. Rudski LG, Lai WW, Afilalo J, Hua L, Handschumacher MD, Chandrasekaran K, Solomon SD, Louie EK, Schiller NB. Guidelines for the echocardiographic assessment of the right heart in adults: a report from the American Society of Echocardiography endorsed by the European Association of Echocardiography, a registered branch of the European Society of Cardiology, and the Canadian Society of Echocardiography. J Am Soc Echocardiogr. 2010, 23:685-713. quiz 786-8

19. Messroghli DR, Rudolph A, Abdel-Aty H, Wassmuth R, Kuhne T, Dietz R, Schulz-Menger J. An open-source software tool for the generation of relaxation time maps in magnetic resonance imaging. BMC Med Imaging. 2010;10:16.

20. Kellman P, Wilson JR, Xue H, Ugander M, Arai AE. Extracellular volume fraction mapping in the myocardium, part 1: evaluation of an automated method. J Cardiovasc Magn Reson. 2012;14:63.

21. Loffredo FS, Nikolova AP, Pancoast JR, Lee RT. Heart failure with preserved ejection fraction: molecular pathways of the aging myocardium. Circ Res. 2014; 115:97-107.

22. Rommel KP, von Roeder M, Latuscynski K, Oberueck C, Blazek S, Fengler K, Besler C, Sandri M, Lucke C, Gutberlet M, Linke A, Schuler G, Lurz P. Extracellular volume fraction for characterization of patients with heart failure and preserved ejection fraction. J Am Coll Cardiol. 2016;67:1815-25.

23. Su MY, Lin LY, Tseng YH, Chang CC, Wu CK, Lin JL, Tseng WY. CMR-verified diffuse myocardial fibrosis is associated with diastolic dysfunction in HFpEF. JACC Cardiovasc Imaging. 2014;7:991-7.

24. Kitzman DW, Shah SJ. The HFpEF obesity phenotype: the elephant in the room. J Am Coll Cardiol. 2016;68:200-3.

25. From AM, Scott CG, Chen HH. The development of heart failure in patients with diabetes mellitus and pre-clinical diastolic dysfunction a populationbased study. J Am Coll Cardiol. 2010;55:300-5.

26. Caughey MC, Avery CL, Ni H, Solomon SD, Matsushita K, Wruck LM, Rosamond WD, Loehr LR. Outcomes of patients with anemia and acute decompensated heart failure with preserved versus reduced ejection fraction (from the ARIC study community surveillance). Am J Cardiol. 2014; 114:1850-4.
27. Gupta DK, Shah AM, Castagno D, Takeuchi M, Loehr LR, Fox ER, Butler KR, Mosley TH, Kitzman DW, Solomon SD. Heart failure with preserved ejection fraction in African Americans: the ARIC (atherosclerosis risk in communities) study. JACC Heart Fail. 2013;1:156-63.

28. Lindman BR, Davila-Roman VG, Mann DL, McNulty S, Semigran MJ, Lewis GD, de las Fuentes L, Joseph SM, Vader J, Hernandez AF, Redfield MM. Cardiovascular phenotype in HFpEF patients with or without diabetes: a RELAX trial ancillary study. J Am Coll Cardiol. 2014;64:541-9.

29. von Haehling S, van Veldhuisen DJ, Roughton M, Babalis D, de Boer RA, Coats AJ, Manzano L, Flather M, Anker SD. Anaemia among patients with heart failure and preserved or reduced ejection fraction: results from the SENIORS study. Eur J Heart Fail. 2011;13:656-63.

30. Berry C, Poppe KK, Gamble GD, Earle NJ, Ezekowitz JA, Squire IB, McMurray JJ, McAlister FA, Komajda M, Swedberg K, Maggioni AP, Ahmed A, Whalley GA, Doughty RN, Tarantini L. Prognostic significance of anaemia in patients with heart failure with preserved and reduced ejection fraction: results from the MAGGIC individual patient data meta-analysis. QJM. 2016;109:377-82.

31. Vazir A, Solomon SD. Management strategies for heart failure with preserved ejection fraction. Heart Fail Clin. 2014;10:591-8.

32. Shah SJ, Katz DH, Selvaraj S, Burke MA, Yancy CW, Gheorghiade M, Bonow RO, Huang CC, Deo RC. Phenomapping for novel classification of heart failure with preserved ejection fraction. Circulation. 2015;131:269-79.
Ready to submit your research? Choose BMC and benefit from:

- fast, convenient online submission

- thorough peer review by experienced researchers in your field

- rapid publication on acceptance

- support for research data, including large and complex data types

- gold Open Access which fosters wider collaboration and increased citations

- maximum visibility for your research: over $100 \mathrm{M}$ website views per year

At BMC, research is always in progress.

Learn more biomedcentral.com/submissions 